This item was submitted to Loughborough's Research Repository by the author.

Items in Figshare are protected by copyright, with all rights reserved, unless otherwise indicated.

\title{
Size-dependent crystal plasticity: from micro-pillar compression to bending
}

PLEASE CITE THE PUBLISHED VERSION

http://dx.doi.org/10.1016/j.mechmat.2016.06.002

\section{PUBLISHER}

(c) Crown copyright. Published by Elsevier

\section{VERSION}

AM (Accepted Manuscript)

\section{PUBLISHER STATEMENT}

This work is made available according to the conditions of the Creative Commons Attribution-NonCommercialNoDerivatives 4.0 International (CC BY-NC-ND 4.0) licence. Full details of this licence are available at: https://creativecommons.org/licenses/by-nc-nd/4.0/

\section{LICENCE}

CC BY-NC-ND 4.0

\section{REPOSITORY RECORD}

Liu, Qiang, Anish Roy, and Vadim V. Silberschmidt. 2019. "Size-dependent Crystal Plasticity: From Micro-pillar Compression to Bending". figshare. https://hdl.handle.net/2134/21833. 


\title{
Size-dependent crystal plasticity: from micro-pillar compression to
}

\section{bending}

Qiang Liu, Anish Roy ${ }^{*}$, Vadim V. Silberschmidt

Wolfson School of Mechanical, Electrical and Manufacturing Engineering, Loughborough University, LE11 3TU, UK

\begin{abstract}
Size-dependent crystal plasticity of metal single crystals is investigated using finiteelement method based on a phenomenological crystal-plasticity model, incorporating both first-order and second-order effects. The first-order effect is independent of the nature of the loading state, and described by three phenomenological relationships based on experimental results. The second-order effect is considered in terms of storage of geometrically necessary dislocations, affected significantly by the loading state. The modelling approach is shown to capture the influence of loading conditions on the sample size effect observed in compression and bending experiments. A modelling study demonstrates the subtleness and importance of accounting for first-order and second-order effects in modelling crystalline materials in small length-scales.
\end{abstract}

\section{Introduction}

It is well known that metallic single crystals at the micron and submicron scale exhibit different mechanical behaviour in comparison to its bulk counterpart. In almost all experimental studies, the phenomenon of 'smaller is stronger' has been observed (see e.g. (Greer and De Hosson, 2011). From a classical standpoint, the sample size effect is typically

\footnotetext{
"Corresponding author.

E-mail address:A.Roy3@lboro.ac.uk
} 
described by a power-law relationship (similar to the Hall-Petch effect), $\sigma_{f}=\sigma_{0}+K d^{-n}$ or $\sigma_{f}=K d^{-n}$, where $\sigma_{f}$ is the measured flow stress, $d$ is the characteristic sample size, and $\sigma_{0}, K$ and $n$ are experimentally fitting parameters (Hug et al., 2015). For different single crystals, experimental results obtained by micro-pillar compression show that $n$ is typically in the range of $0.6 \sim 1.0$ for FCC metals and 0.5 or less for BCC metals (Tarleton et al., 2015). Experimental data for HCP metals indicate that $n$ is $\sim 0.5$ for prismatic slip in Ti (Sun et al., 2011), 0.8 (Ye et al., 2011) or 0.4 (Byer and Ramesh, 2013) for basal slip and 0.2 for pyramidal slip in Mg (Byer and Ramesh, 2013). Apart from the size-dependent strengthening effect, a size-dependent softening was also reported when a reverse loading was applied on the cantilever-beam of single-crystal copper (Demir and Raabe, 2010).

Although the sample size effect of single crystal is experimentally described by the power-law relationship, the underlying physical mechanism driving size effects is still debated. Geers et al. (Geers et al., 2006) categorized the size effect in polycrystalline metals into (i) intrinsically first-order effect, which was considered to cover all effects resulting from the nature of microstructure and (ii) second-order effect ${ }^{\dagger}$, which was considered to result from gradients of deformation (strain gradient, slip gradient, etc.). We adopt a similar classification in this paper for single-crystal metals. In a single-crystal sample, as there is no microstructural feature related to grain boundary and the heterogeneity of grains, the firstorder effect can be determined from several dislocation-mediated mechanisms, including source-limitation, dislocation starvation and source-truncation hardening mechanisms, amongst others (El-Awady, 2015; Kiener et al., 2006). The second-order effect mainly originates from inhomogeneous plastic deformation or slip gradient in a single-crystal sample (e.g. due to bending).

\footnotetext{
${ }^{\dagger}$ Not to be confused with higher order (gradient) theories
} 
Due to the different underlying physical mechanisms, a quantitative difference may be observed for the sample size effect measured in different loading conditions. For example, when the power-law relationship is employed to characterize the sample size effect in a $\mathrm{Cu}$ single crystal, the measured $n$ is about 0.4 for micro-pillar compression (Kiener et al., 2006) and 0.8 for cantilever beam experiments (Motz et al., 2005). In Ti, the value of $K$ for prismatic slip is about $131 \mathrm{~Pa}-\mathrm{m}$ from micro-pillar compression tests (Sun et al., 2011) but 354 Pa-m from cantilever-beam experiments (Gong and Wilkinson, 2011), although the values of $\sigma_{0}$ and $n$ are comparable for the two loading conditions. These experimental data indicates that the sample size effect in bending, due to the coexistence of first-order and second-order effects (i.e. externally imposed stress/strain gradients), is more pronounced than that in uniaxial compression where first-order effect dominate. Here, the dependence of size effect on loading conditions cannot be depicted by the popular power-law relationship. Moreover, the experimental results of Maass et al. (Maass et al., 2009) indicate that the power-law relationship based on flow stress could overestimate the true size effect due to the influences of boundary constraints on the measured hardening behaviour. Consequently, the simplified power-law relationship is incomplete (or incorrect) in describing size effect, especially when both first-order and second-order effects dominate.

To overcome the drawback of the power-law relationship approach, crystal-plasticity (CP) modelling was employed to help extract the nature of size effect in single-crystal metal (Gong and Wilkinson, 2011; Raabe et al., 2007). From a modelling perspective, the first-order effect is typically modelled using conventional CP based constitutive models, which suffer from several short comings. The second-order effect may be described by strain-gradient-based model (Geers et al., 2006). In a CP modelling framework, the second-order effect was general modelled as plastic strain gradient (Roters et al., 2010). The non-uniform plastic deformation was generally associated with the storage of geometrically necessary dislocations (GNDs) in 
contrast to statistically stored dislocations (SSDs) that is considered independent of plastic strain gradient (Faghihi and Voyiadjis, 2012). Size-dependent work-hardening will become significant when the storage of GNDs is comparable to SSDs, leading to the second-order effect. Such a strain-gradient effect, associated with GNDs, has been introduced into CP model by two approaches. One is based on high-order CP theory that requires additional boundary conditions which are difficult to determine physically (Reuber et al., 2014). The other being a lower-order strain-gradient $\mathrm{CP}$ theory, where the storage of GNDs are introduced into the evolution of SSDs and calculation of slip-system resistance (Ma et al., 2006).

Our primary goal is to characterize both the first-order and second-order effects in smallscale single crystals using a CP theory. Three phenomenological relationships are proposed to describe the first-order effect based on micro-pillar compression experiments and discrete dislocation dynamics (DDD) simulation studies. A low-order strain-gradient CP approach is adopted to introduce the second-order effect in the current study. Contributions of the secondorder effect, in addition to the first-order one, are estimated from cantilever-beam experimental data. Numerical studies show that the proposed modelling framework is capable to characterise size effects under macroscopically homogeneous and in-homogeneous loading states.

The paper is organized as follows: in Section 2, a self-contained description of the governing relations of proposed size-dependent $\mathrm{CP}$ model is presented. Section 3 comprises a finite-element modelling strategy implemented in a general commercial finite element software package ABAQUS. In Section 4, results of the implementation are presented and discussed. The paper ends with some concluding remarks in Section 5. 


\section{Constitutive description of first-order and second-order effects}

In this section, a phenomenological size-dependent crystal plasticity (SDCP) model is proposed, which accounts for the first-order and second-order effects of crystalline metals. Standard notations are adopted here: scalars are in italics, vectors and tensors are represented with lower-case and upper-case bold letters.

First, for completeness, the classical CP theory adopted in this study is reviewed. Deformation gradient $\mathbf{F}$, can be decomposed into the elastic and plastic parts (Roters et al., 2010), as,

$$
\mathbf{F}=\mathbf{F}_{\mathrm{e}} \mathbf{F}_{\mathrm{p}}
$$

where the subscripts ' $e$ ' and ' $p$ ' denote the elastic and plastic parameters, respectively. The multiplicative decomposition is non-unique. By applying the product rule of differentiation, one can obtain the rate of the total deformation gradient $\dot{\mathbf{F}}$ :

$$
\dot{\mathbf{F}}=\dot{\mathbf{F}}_{\mathrm{e}} \mathbf{F}_{\mathrm{p}}+\mathbf{F}_{\mathrm{e}} \dot{\mathbf{F}}_{\mathrm{p}}
$$

Therefore, the velocity gradient $\mathbf{L}$ can be introduced following its definition $\mathbf{L}=\dot{\mathbf{F F}}^{-\mathbf{1}}$, as,

$$
\mathbf{L}=\dot{\mathbf{F}}_{\mathrm{e}} \mathbf{F}_{\mathrm{e}}^{-1}+\mathbf{F}_{\mathrm{e}}\left(\dot{\mathbf{F}}_{\mathrm{p}} \mathbf{F}_{\mathrm{p}}^{-1}\right) \mathbf{F}_{\mathrm{e}}^{-1}=\mathrm{L}_{\mathrm{e}}+\mathbf{L}_{\mathrm{p}}
$$

It is assumed that the plastic velocity gradient, $\mathbf{L}_{\mathbf{p}}$, is induced by shearing on each slip system. Hence, $\mathbf{L}_{\mathbf{p}}$ is formulated as the sum of the shear rates on all slip systems, i.e.

$$
\mathbf{L}_{\mathbf{p}}=\sum_{\alpha=1}^{N} \dot{\gamma}^{(\alpha)} \mathbf{s}^{(\alpha)} \otimes \mathbf{m}^{(\alpha)}
$$

where, $\dot{\gamma}^{(\alpha)}$ is the shear slip rate on the slip system $\alpha, N$ is the total number of slip systems, and unit vectors $\mathbf{s}^{(\alpha)}$ and $\mathbf{m}^{(\alpha)}$ define the slip direction and the normal to the slip plane in the deformed configuration, respectively. Furthermore, the velocity gradient can be expressed in terms of a symmetric rate of stretching $\mathbf{D}$ and an antisymmetric rate of spin $\mathbf{W}$, as,

$$
\mathbf{L}=\mathbf{D}+\mathbf{W}=\left(\mathbf{D}_{\mathrm{e}}+\mathbf{W}_{\mathrm{e}}\right)+\left(\mathbf{D}_{\mathrm{p}}+\mathbf{W}_{\mathrm{p}}\right) \text {. }
$$


Using Eq. (3) and (4), it can be deduced

$$
\mathbf{D}_{\mathbf{e}}+\mathbf{W}_{\mathbf{e}}=\dot{\mathbf{F}}_{\mathbf{e}} \mathbf{F}_{\mathbf{e}}^{-\mathbf{1}}, \mathbf{D}_{\mathbf{p}}+\mathbf{W}_{\mathbf{p}}=\sum_{\alpha=1}^{N} \dot{\gamma}^{(\alpha)} \mathbf{s}^{(\alpha)} \otimes \mathbf{m}^{(\alpha)}
$$

Following the work of Huang (Huang, 1991), the constitutive law is expressed as the relationship between the elastic part of the symmetric rate of stretching, $\mathbf{D}_{\mathbf{e}}$, and the Jaumann rate of Cauchy stress, $\stackrel{\nabla}{\sigma}$, i.e.

$$
\stackrel{\nabla}{\sigma}+\sigma\left(I: D_{e}\right)=C:\left(D-D_{p}\right)
$$

where, $\mathbf{I}$ is the second-order unit tensor, $\mathbf{C}$ is the fourth order, possibly anisotropic, elastic stiffness tensor. The Jaumann stress rate is expressed as

$$
\stackrel{\nabla}{\sigma}=\dot{\sigma}-\mathbf{W}_{\mathrm{e}} \boldsymbol{\sigma}+\boldsymbol{\sigma} \mathbf{W}_{\mathrm{e}}
$$

On each slip system, the resolved shear stress, $\tau^{(\alpha)}$, is expressed by Schmid law,

$$
\tau^{(\alpha)}=\left(\mathbf{s}^{(\alpha)} \otimes \mathbf{m}^{(\alpha)}\right): \boldsymbol{\sigma}
$$

The relationship between the shear rate, $\dot{\gamma}^{(\alpha)}$, and the resolved shear stress, $\tau^{(\alpha)}$, on the slip system, $\alpha$, is expressed by the power law proposed by Hutchinson (Hutchinson, 1976):

$$
\dot{\gamma}^{(\alpha)}=\dot{\gamma}_{0}\left|\frac{\tau^{(\alpha)}}{g^{(\alpha)}}\right|^{n} \operatorname{sgn}\left(\tau^{(\alpha)}\right)
$$

where, $\dot{\gamma}_{0}$ is the reference shear rate, $g^{(\alpha)}$ is the slip resistance and $n$ is the rate sensitivity parameter. Next, the model is developed to account for the first-order and second-order effects, which are introduced into the calculation of $g^{(\alpha)}$.

\subsection{First-order effect}

In the absence of strain gradient, it is generally accepted that $g^{(\alpha)}$ is determined by the content of statistically stored dislocations (SSDs) in the component. For a single crystal at macro-scale, nucleation of dislocations is relatively easy (El-Awady, 2015) due to the 
geographic abundance of nucleation sites. Thus, slip resistance in the macro-scale can be described by the empirical Taylor model (El-Awady, 2015). However, at smaller length scales, a higher nucleation stress is required following source-limitation strengthening mechanism (Kiener et al., 2006; Tarleton et al., 2015). Besides, the existence of single-arm sources also results in a difficulty of operating dislocation sources based on dislocationtruncation mechanism (Parthasarathy et al., 2007). Consequently, these strengthening mechanisms will lead to higher initial yield stress, which has been verified by the Laue diffraction analysis (Maass et al., 2009). To capture this effect phenomenologically, a sizedependent term is introduced into the expression of $g^{(\alpha)}$, similar to the work of Gong et al. (Gong and Wilkinson, 2011),

$$
\begin{aligned}
& g^{(\alpha)}=\tau_{D}+\tau_{S}, \\
& \tau_{D}=\chi \mu b \sqrt{\sum_{\beta} \rho_{S S D}^{(\beta)}}, \tau_{S}=\tau_{0} \frac{D_{1}^{0}}{d},
\end{aligned}
$$

where, $\tau_{D}$ is the slip strength contribution from dislocation interactions and $\tau_{S}$ accounts for the increase in the nucleation stress caused by source-limitation strengthening and dislocation-truncation mechanisms. The hardening coefficient is presented by $\chi$, and $\mu$ and $b$ are the shear modulus and Burgers vector, respectively. The characteristic size of a single crystal sample is introduced by $d ; \tau_{0}$ is the initial slip resistance independent of sample size. $D_{1}^{0}$ is the reference sample size, and the nucleation stress increases significantly when sample size decreases to the magnitude of $D_{1}^{0}$. The initial slip resistance $g_{0}^{(\alpha)}$ can therefore be expressed as

$$
\begin{aligned}
& g_{0}^{(\alpha)}=\tau_{0}+\tau_{S}=\tau_{0}\left(1+\frac{D_{1}^{0}}{d}\right), \\
& \tau_{0}=\chi \mu b \sqrt{\sum_{\beta} \rho_{S S D, 0}^{(\beta)}}, \tau_{S}=\chi \mu b \sqrt{\sum_{\beta} \rho_{S S D, 0}^{(\beta)}} \times\left(\frac{D_{1}^{0}}{d}\right) .
\end{aligned}
$$


Here, the initial dislocation density $\rho_{S S D, 0}^{(\beta)}$ can be calculated by $\tau_{0}$. Thus, the size-dependent term, $\tau_{S}$, only contributes to the magnitude of the initial yield stress.

As mentioned earlier, the source-limitation, dislocation-starvation and sourcetruncation hardening mechanisms were widely adopted to account for intrinsically first-order effect (El-Awady, 2015; Kiener et al., 2006). The source-limitation and source-truncation hardening mechanisms lead to the increase of dislocation nucleation stress (Kiener et al., 2006; Parthasarathy et al., 2007; Tarleton et al., 2015). In the meantime, the dislocationstarvation and source-truncation mechanism have an effect on dislocation multiplication (Parthasarathy et al., 2007; Tang et al., 2007). According to the dislocation-starvation hardening mechanism, due to limited mobile dislocations in a small-scaled single-crystal component, an increase in the flow stress is expected (Parthasarathy et al., 2007). Additionally, DDD simulations show that the existence of single-arm sources induce a smaller number of long dislocation segments and junctions at smaller scales (Tang et al., 2007). Micropillar compression studies in $\mathrm{Cu}$ showed that the hardening rate increased with smaller sample size (Kiener et al., 2011). Therefore, it can be inferred that the hardening behaviour will be affected by limited mobile dislocations and initial single-arm sources in the component. To describe the increasing hardening rate with decreasing size, we assume the hardening coefficient $\chi$ in Eq.(11) and (12) to be size-dependent, as,

$$
\chi=\chi_{0}\left(1+\frac{D_{1}^{0}}{d}\right)
$$

where $\chi_{0}$ is the hardening coefficient at macro-scale. Equation (13) implies that for a fixed magnitude of dislocation density the generation and multiplication of dislocations require higher stress level for a smaller sized single crystal sample. Note that $\tau_{0}$ is independent of 
size, and hence, the initial density of SSDs decreases with decreasing size, which is similar to the phenomenological model proposed in the work of El-Awady (El-Awady, 2015).

DDD simulations also demonstrate that there is a limit to the amount of dislocations, which can be physically sustained in small sized crystalline components (Deshpande et al., 2005; Kiener et al., 2011). That is, the dislocation content in components will saturate to a value, which is observed to depend on sample size. The evolution of SSDs is described using a classical phenomenological equation as follows,

$$
\dot{\rho}_{S S D}^{(\alpha)}=\left(k_{1} \sqrt{\sum_{\beta} \rho_{S S D}^{(\beta)}}-k_{2} \rho_{S S D}^{(\alpha)}\right)\left|\dot{\gamma}^{(\alpha)}\right|,
$$

where $k_{1}$ and $k_{2}$ are the material parameters, representing the generation and annihilation of dislocations, respectively. When the balance between generation and annihilation is achieved (i.e. $\dot{\rho}_{S S D}^{(\alpha)}=0$, the dislocation densities, $\rho_{S S D, \text { sat }}^{(\alpha)}$, will saturate. It can be deduced that this is proportional to $\left(k_{1} / k_{2}\right)^{2}$ when $\dot{\rho}_{S S D}^{(\alpha)}=0$. In the macro-scale, $\rho_{S S D, \text { sat }}^{(\alpha)}$ is fixed; however, for sample sizes in the micron and submicron scale, dislocations have an increase propensity to escape from a free surface (this is expected to increase with a decrease in the sample size). Therefore, $\rho_{S S D, s a t}^{(\alpha)}$ will decrease as the sample size decreases, as verified by DDD simulations (Deshpande et al., 2005; Kiener et al., 2011). In other words, the parameter $k_{2}$, representing the annihilation of dislocation, should be size-dependent to reflect dislocations loss from the free surface. This is expressed as

$$
k_{2}=k_{2}^{0}\left[1+\left(\frac{D_{2}^{0}}{d}\right)^{\eta}\right]
$$

where $k_{2}^{0}$ is the annihilation coefficient of dislocations at macro-scale. The reference sample size, representing dislocations escape from a free surface, is defined as $D_{2}^{0}$, and $\eta$ is dependent on sample geometry. The equation implies that, with sample sizes comparable to 
$D_{2}^{0}$, the magnitude of saturated dislocation density becomes size dependent. When the sample size decreases to the magnitude of $D_{2}^{0}$, the crystal contains a relatively high number of dislocation sources producing a high mobile dislocation density at yield. Therefore, $D_{2}^{0}$ should be larger than $D_{1}^{0}$ that defines the scale of the crystal with limited source or mobile dislocations. A similar conclusion was also drawn in the literature (El-Awady, 2015). From Eq. (14), it can be inferred that $\rho_{S S D, s a t}^{(\alpha)}$ is proportional to $\left(k_{1} / k_{2}\right)^{2}$. We assume that $\rho_{S S D, s a t}^{(\alpha)}$ decreases with the volume of a single-crystal sample, $V$, i.e.

$$
\rho_{S S D, s a t}^{(\alpha)} \propto\left(\frac{k_{1}}{k_{2}}\right)^{2} \propto V
$$

In general, $V$ is proportional to $\sim d^{2}$ for a sample with large aspect ratio and $\sim d^{3}$ for components with a small aspect ratio. Therefore, Eq. (16) indicates that the value of $\eta$ should be in the range of 1.0 1.5 for a typical sample geometry.

\subsection{Second-order effect}

During component deformation, strain gradients are generated in the material volume, which manifest as GNDs. Thus, the contribution of GNDs to slip resistance and the evolution of SSDs should be considered (van Beers et al., 2015). Consequently, the net scalar GND density, $\rho_{G N D}^{(\beta)}$, is introduced into Eq. (11) and (14), as

$$
\begin{aligned}
& g^{(\alpha)}=\chi \mu b \sqrt{\sum_{\beta}\left(\rho_{S S D}^{(\beta)}+\rho_{G N D}^{(\beta)}\right)}+\tau_{0} \frac{D_{r e f, 1}}{d} \text { and } \\
& \dot{\rho}_{S S D}^{(\alpha)}=\left(k_{1} \sqrt{\sum_{\beta}\left(\rho_{S S D}^{(\beta)}+\rho_{G N D}^{(\beta)}\right)}-k_{2} \rho_{S S D}^{(\alpha)}\right)\left|\dot{\gamma}^{(\alpha)}\right| .
\end{aligned}
$$

Here, the GND density is defined as vector 'density' following the work of Ma et al. (Ma et al., 2006). The rate of GND density evolution, $\dot{\boldsymbol{\rho}}_{\mathrm{GND}}^{(\boldsymbol{\alpha})}$, is represented as 


$$
\dot{\boldsymbol{\rho}}_{\mathbf{G N D}}^{(\boldsymbol{\alpha})}=\frac{1}{b}\left(\nabla \times\left(\dot{\gamma}^{(\alpha)} \mathbf{F}_{\mathbf{P}}^{\mathbf{T}} \mathbf{m}^{(\alpha)}\right) .\right.
$$

The net scalar GND density is defined as $\rho_{G N D}^{(\alpha)}=\left|\boldsymbol{\rho}_{\mathbf{G N D}}^{(\boldsymbol{\alpha})}\right|$. As per the typical interpretation of GND density, an increase in the gradient of plastic deformation leads to an increase of GND density. Here, we make a crucial point that there is a threshold to the dislocation density, which can be sustained in a component. In other words, there is a physical limit to the saturated GND density under deformations. Experimental results of Huang et al. (Huang et al., 2006) support the fact that GND density ultimately attains a saturated value, $\rho_{G N D}^{\max }$, which can be used to redefine Eq. (19) as

$$
\dot{\boldsymbol{\rho}}_{\mathbf{G N D}}^{(\boldsymbol{\alpha})}= \begin{cases}\dot{\boldsymbol{\rho}}_{\mathbf{G N D}}^{(\boldsymbol{\alpha})}=\frac{1}{b}\left(\nabla \times\left(\dot{\gamma}^{(\alpha)} \mathbf{F}_{\mathbf{P}}^{\mathbf{T}} \mathbf{m}^{(\alpha)}\right),\right. & \text { if } \rho_{G N D}^{(\alpha)} \leq \rho_{G N D}^{\max } \\ \mathbf{0}, & \text { otherwise }\end{cases}
$$

\section{Finite-element model}

The phenomenological SDCP model proposed in Section 2 was implemented in the commercial finite-element (FE) code ABAQUS/Standard by employing the user subroutine UMAT. The aim is to demonstrate effectiveness of the model in capturing the first-order and second-order effects in copper single crystal based on a micro-pillar compression experiment of Kiener et al. (Kiener et al., 2009) and a cantilever-beam experiment of Motz et al. (Motz et al., 2005).

The calculation of a strain gradient is realized with the use of a C3D8 element in ABAQUS, similar to the method outlined in (Ma et al., 2006). The shear modulus and Burgers vector are $48.5 \mathrm{GPa}$ and $0.256 \mathrm{~nm}$, respectively. The initial GND density was set as zero (since GNDs are typically associated with internal strain gradients that are absent in an unloaded sample), while the initial SSD density is calculated based on the size-independent initial slip resistance $\tau_{0}$. Other model parameters are listed in Table 1 for single crystal copper. In our studies, the characteristic sample size was chosen as the edge length of a 
square cross-section for micro-pillar compression sample, and the sample thickness for a cantilever beam.

Figure 1 shows a schematic of the FE models used to describe the micro-pillar and cantilever beam experiment, in which the crystal orientation and sample geometry is imposed following these experiments (Motz et al., 2005). A cross-section of the micro-pillar is assumed to be square and constant along its height. For the cantilever-beam, its effective width, thickness and length are denoted by $W, H$ and $L$, respectively (Fig. 1(b)). Loading of cantilever beam was realized with a spherical indenter as in the experiment. The indenter's radius was $2.0 \mu \mathrm{m}$ for all simulations (our simulations show that the perceived indenter/reaction load is independent of the indenter radius).

The micro-pillar compression experiment (Kiener et al., 2009) referred in this paper considers a sample with a constant cross-sectional area and length-width aspect ratio of $\sim 2.0$. We note that instability in the deformation during micro-pillar compression can be restrained since friction is present in experiments (Raabe et al., 2007). Consequently, for micro-pillar compression, the displacement boundary conditions prescribed are shown in Fig. 1(a); they are

$$
\left.u_{X}\right|_{B_{1}}=0,\left.u_{Y}\right|_{B_{2}}=0,\left.u_{Z}\right|_{B_{3}}=0
$$

where, $B_{1}, B_{2}$ and $B_{3}$ are the bottom, left and back face of the pillar as per the schematic in Fig. 1. The applied displacement on the top surface, $\bar{U}_{Y}$, is determined by the applied strain, $\bar{\varepsilon}_{Y}$, as

$$
\bar{U}_{Y}=L_{H} \bar{\varepsilon}_{Y}
$$

where $L_{H}$ is the height of the micro-pillar. Displacement boundary conditions for the cantilever beam experiment are shown in Fig. 1(b), which are

$$
\left.u_{X}\right|_{\Gamma_{1}}=0
$$




$$
\left.u_{Y}\right|_{\Gamma_{2}}=0,\left.u_{Z}\right|_{\Gamma_{2}}=0,
$$

where, $\Gamma_{1}$ and $\Gamma_{2}$ are the left and bottom face as per the schematic in Fig. 1(b). To facilitate a quantitative estimation of sample size effect in bending, the effective flow stress of cantilever beam $\sigma_{B, f}$ was calculated following the definition as in the experiment (Gong and Wilkinson, 2011; Motz et al., 2005),

$$
\sigma_{B, f}=\frac{4 F_{\max } L}{W H^{2}},
$$

where $F_{\max }$ represents the maximum load in bending of the cantilever beam. In the numerical study, about 25,000 elements were used to mesh the cantilever beam, after an exhaustive mesh-convergence study.
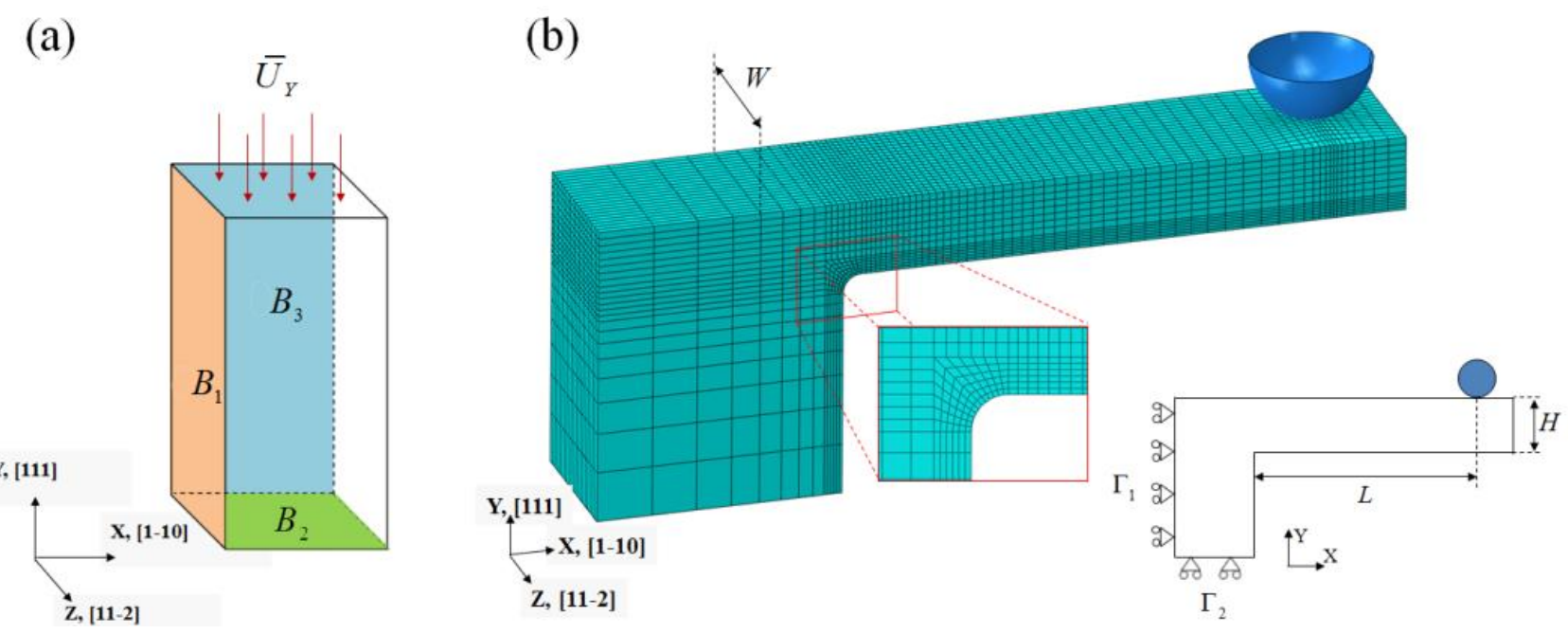

Fig. 1 Schematic of FE models for micro-pillar compression (a) and cantilever beam (b)

Table 1 Model parameters of single crystal copper

\begin{tabular}{llll}
\hline Parameter & Definition & Value & Unit \\
\hline$\dot{\gamma}_{0}$ & Reference shear rate & 0.001 & $\mathrm{~s}^{-1}$ \\
$n$ & Rate-sensitivity parameter & 50 & - \\
$\tau_{0}$ & Size-independent initial slip resistance & 16.0 & $\mathrm{MPa}$ \\
\hline
\end{tabular}




\begin{tabular}{llll}
\hline$\chi_{0}$ & Macro-scale hardening coefficient & 0.25 & - \\
$D_{1}^{0}$ & Reference sample size of limited source & 8.5 & $\mu \mathrm{m}$ \\
$k_{1}$ & SSD generation coefficient & 130.0 & $\mu \mathrm{m}^{-1}$ \\
$k_{2}^{0}$ & $\begin{array}{l}\text { Macro-scale SSD annihilation } \\
\text { coefficient }\end{array}$ & 13.3 & - \\
$D_{2}^{0}$ & $\begin{array}{l}\text { Reference sample size of mobile } \\
\text { dislocation escaping }\end{array}$ & 20.0 & $\mu \mathrm{m}$ \\
$\eta$ & Adjustable parameter & 1.2 & - \\
$\rho_{G N D}^{\max }$ & Saturated GND density & 15 & $\mu \mathrm{m}^{-2}$ \\
\hline
\end{tabular}

\section{Results and discussions}

\subsection{Size-independent response of macro-size samples}

First, experimental data for the macro-size copper single crystal are employed to calibrate the developed SDCP model. Compression of a copper single crystal in [011] orientation was reported by Kalidindi and Anand (Kalidindi and Anand, 1993) with diameter of $12.7 \mathrm{~mm}$, and in [111] orientation with diameter of $25.0 \mathrm{~mm}$ by Takeuchi (Takeuchi, 1975). Numerical results show an excellent match with these experimental data for the specific orientations (Fig. 2). As expected, the SDCP model reduces to a conventional crystal plasticity (CCP) model describing the size-independent behaviour of a macro-scale copper single crystal. 


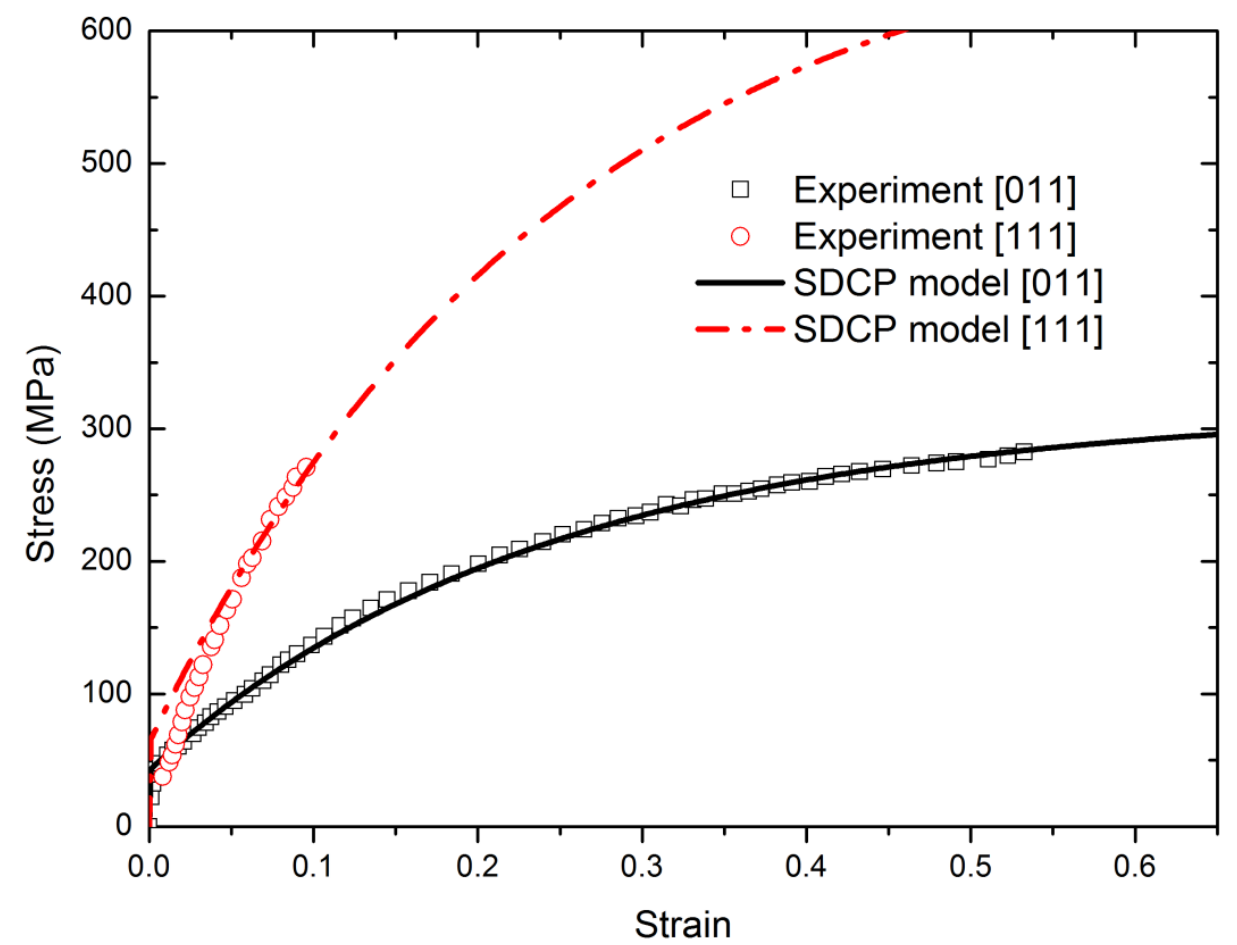

Fig. 2 Comparison of experimental data and simulation results under compression of copper single crystal at macro-scale

\subsection{Size effect in compression}

In the SDCP model, three parameters are used to characterize the first-order effect. For this, the experimental data from compression studies of a square micro-pillar with a constant cross-sectional area of $4.82 \mu m \times 4.82 \mu m$ (Kiener et al., 2009) was employed. The nature of deformation implies that external strain-gradient effect can be eliminated in the compressiontest analysis (in the limit of small-deformations).

Here, we demonstrate the importance of (i) accounting for a size-dependent hardening rate, and, (ii) size-dependence over and above that in CCP models. In Fig. 3, the simulation results obtained with the CCP model (which is size independent) shows a significant difference with experimental data. Such a difference demonstrates the influence of the firstorder effect on small-scale plasticity, which can be summarized as the following features observed with a decrease in the sample size: (1) an increase of the initial yield stress, (2) decrease of flow stress relative to the initial yield stress (weakening of hardening behaviour) 
and (3) an increase in the hardening rate. These observations are based on the micro-pillar compression experiments in single crystal copper (Kiener et al., 2011; Maass et al., 2009). Numerical results from the SDCP model, considering the proposed size-dependent hardening (as in Eq. (13)) and the standard hardening rate (i.e., $\chi=\chi_{0}$ ), are also presented in Fig. 3. The results show that when the standard hardening rate is considered, the hardening modulus is underestimated in the small-strain range but overestimated in the large-strain range when compared to the experimental results. The proposed SDCP model provides a better match with the experimental data. The comparison of the simulation results and the experimental data indicates that the SDCP model is more effective in small-scale plasticity than the widely adopted CCP model. The size-dependent initial yield stress and strain hardening behaviour can be better differentiated by combining the SDCP model and in-situ Laue diffraction analysis as described in the work of Maass et al. (Maass et al., 2009).

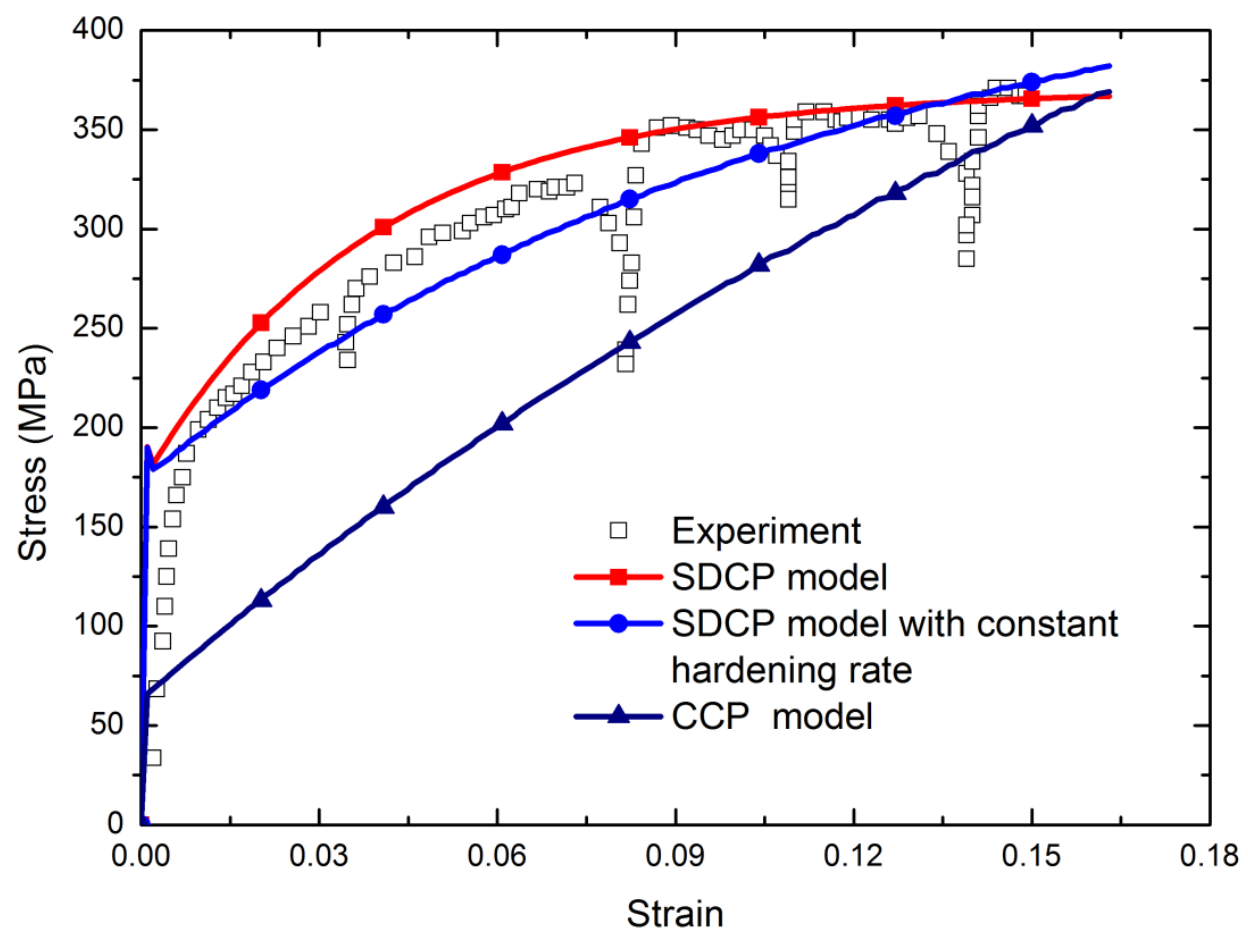

Fig. 3 Comparisons between experimental data and simulation results during compression of copper micro-pillar with square cross-section of $4.82 \mu m \times 4.82 \mu m$ and loading direction along [111] 
Numerical predictions of stress-strain curves for different pillar sizes are shown in Fig. 4. The loading direction is along [111] for all the cases. The importance of accounting for the first-order size-effect can be appreciated when comparing the stress-strain response of a bulk sizes specimen in Fig. 4. Apart from the initial yield stress, the flow stress, $\sigma_{f}$, defined as the stress at $10 \%$ overall strain (Kiener et al., 2009), increases with the decreasing sample size. However, the hardening behaviour becomes less significant in a smaller scale. As shown in Fig. 5, the SDCP model accurately captures the first-order effect for single crystal copper micro-pillars with the edge length in the range of $0.8-8.0 \mu \mathrm{m}$. The first-order effect becomes prominent for the edge length smaller than $5.0 \mu \mathrm{m}$. The experimental data for flow stress measured with cantilever bending experiments are also presented in Fig. 5, demonstrating that the size effect in bending is more significant than that in compression due to the secondorder effect, which will be studied in the following section.

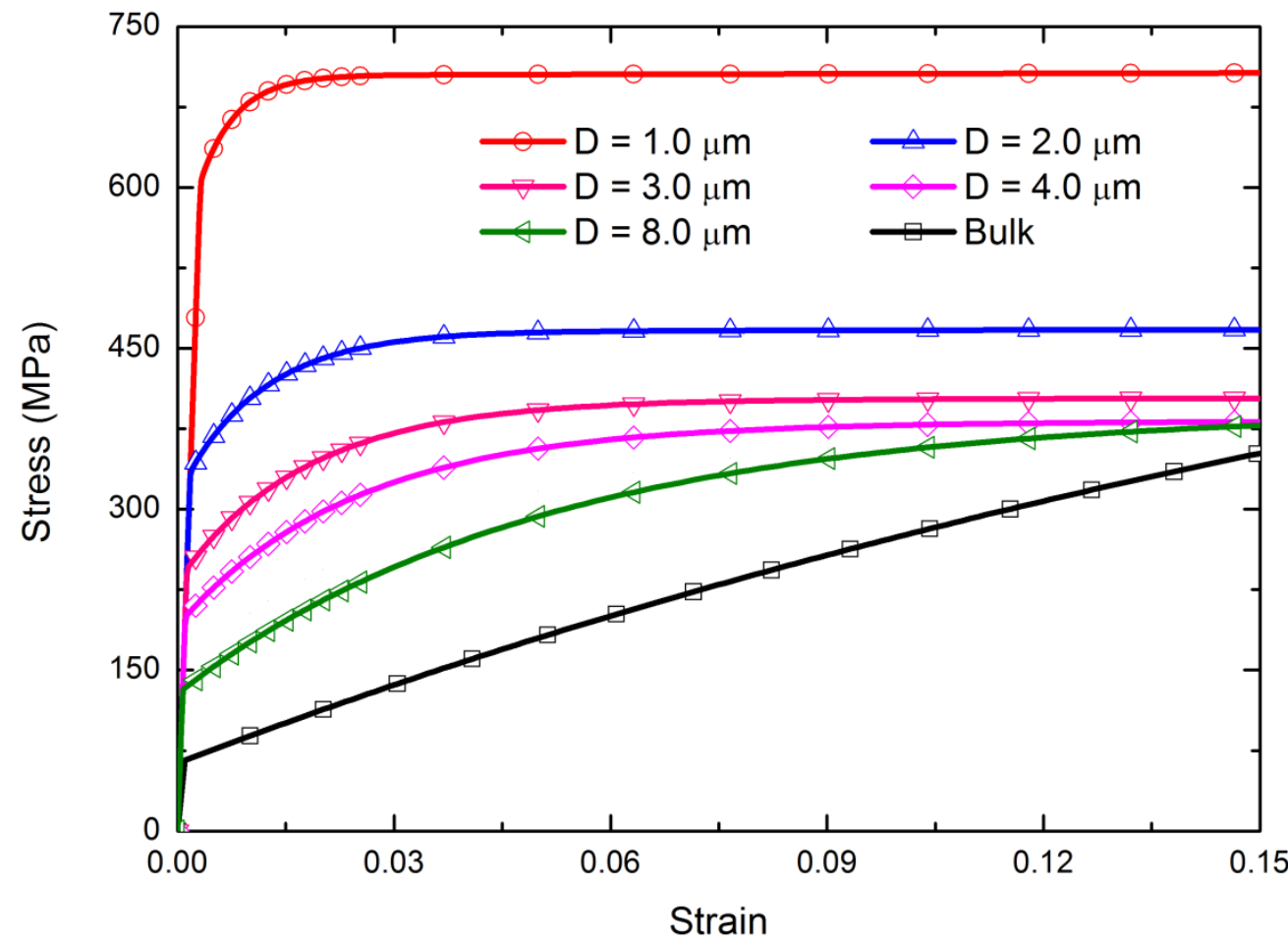

Fig. 4 First-order effect shown by compressive stress-strain curves of single crystal copper with loading direction along [111] ( $D$ is the side length of the square cross-section) 


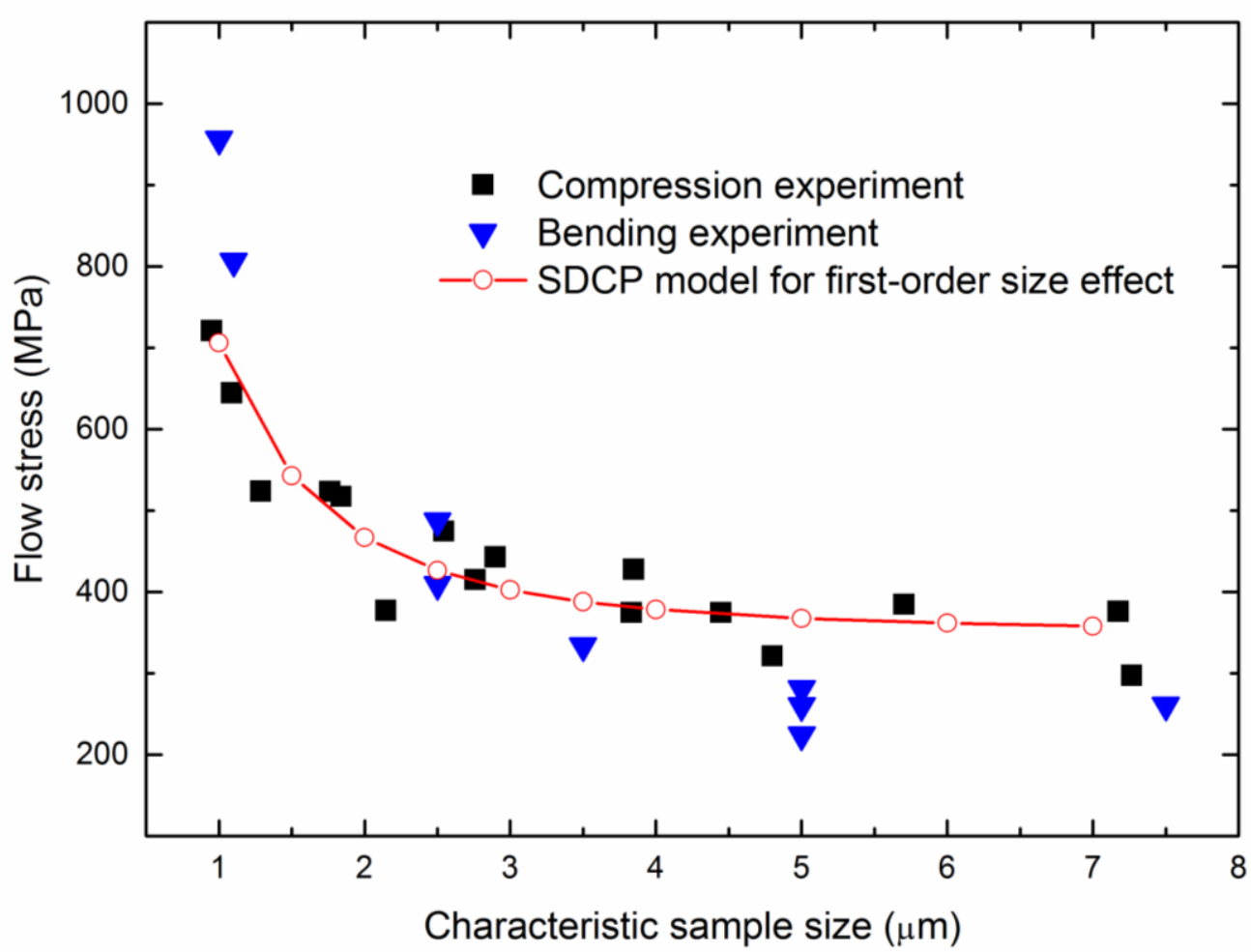

Fig. 5 First-order effect for single crystal copper obtained by micro-pillar compression and micro-cantilever bending: experimental data and simulation results

\subsection{Size effect in bending}

The second-order effect is quantified with the aid of beam bending experiments. In the SDCP model, the saturated GND density was calibrated according to the measured loaddisplacement curves of the cantilever beam with dimensions of $5.0 \mu m \times 5.0 \mu m \times 20.0 \mu m$ (Motz et al., 2005) as shown in Fig. 6. It is obvious that the magnitude of $\rho_{G N D}^{\max }$ affects the hardening behaviour during bending. The load level increases significantly with an increase in $\rho_{G N D}^{\max }$. From our initial numerical study, $\rho_{G N D}^{\max }=15 \mu m^{-2}$ provides a reasonable match with the macroscopic load-displacement response from the experiments (Fig. 6). In comparison, the load calculated for $\rho_{G N D}^{\max }=20 \mu \mathrm{m}^{-2}$ is much larger after displacement of 500nm, indicating 
that GND accumulation is restricted when the strain gradient is relatively large, similar to the conclusion drawn by Huang et al. (Huang et al., 2006).

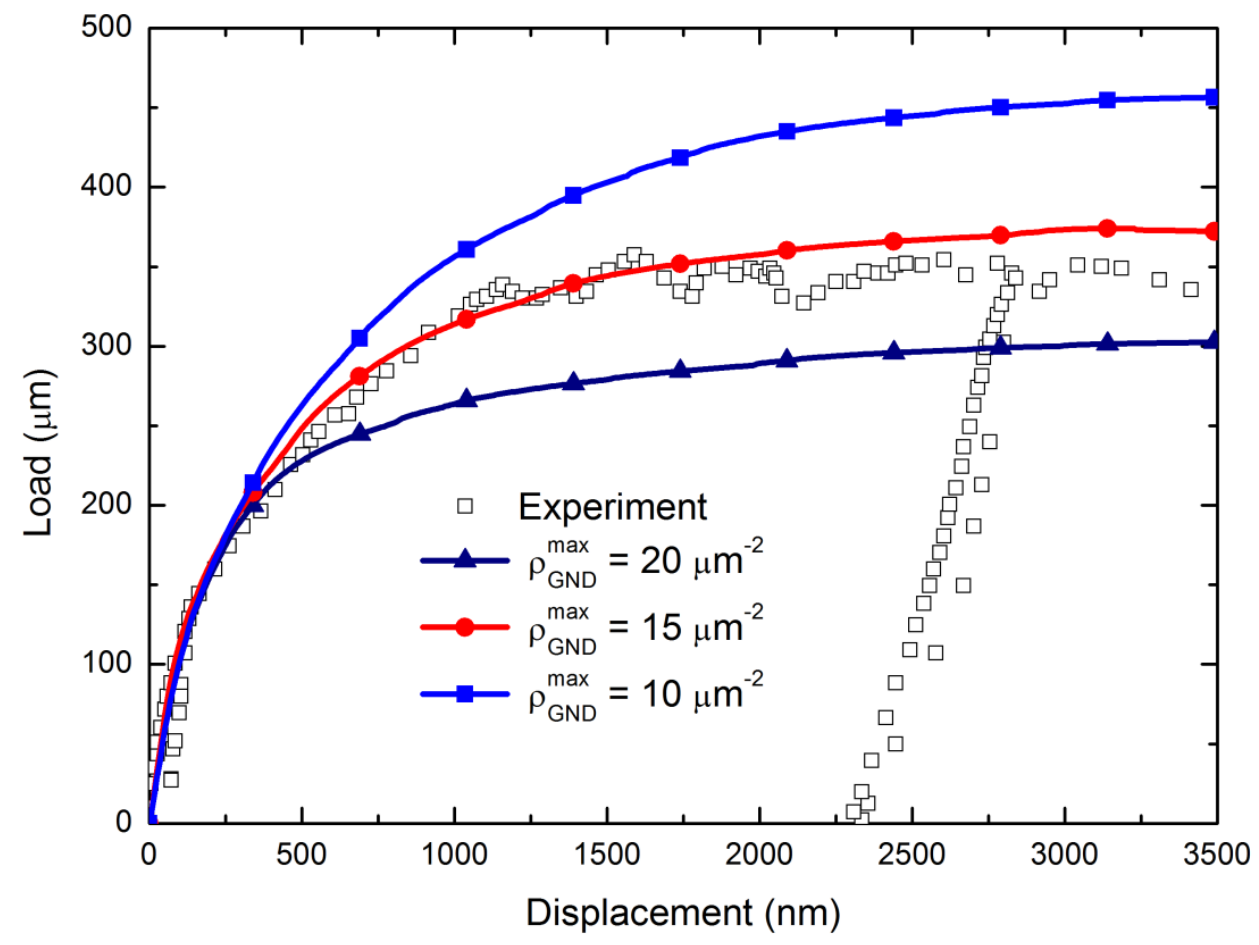

Fig. 6 Effect of GNDs on load-displacement curves of cantilever beam with dimensions of $5.0 \mu m \times 5.0 \mu m \times 20.0 \mu m$

Figure 7(a) shows the comparison of simulation results and experiment data for a cantilever beam with dimensions of $5.0 \mu m \times 2.5 \mu m \times 16.3 \mu m$ (Motz et al., 2005). The results demonstrate the influence of first-order and second-order effects on bending behaviour. The peak-load value obtained with the CCP model is only $15 \%$ of the experimentally measured load value, showing a poor match. Incorporating the first-order effect (i.e. the SDCP model with only the first-order effect) improves predictions when compared to those of the CCP model. However, the peak-load value is $\sim 50 \%$ of the experimentally measured value when the second-order effect is not accounted for. Introducing both first-order and second-order effects as in the full SDCP model shows an excellent correlation with the experimental data. Figure 7(b) shows the distribution of slip resistance on the slip system $[-111]<101>$ corresponding to the loading states (1) and (2) 
in Figure 7(a). The field plots show that the accumulation of GNDs (as in (1) when compared to (2) leads to the significant increase in slip resistance. Thus, the secondorder effect is crucial for small-scale plasticity, especially when modelling loading states, which induce macroscopic strain gradient. 
(a)

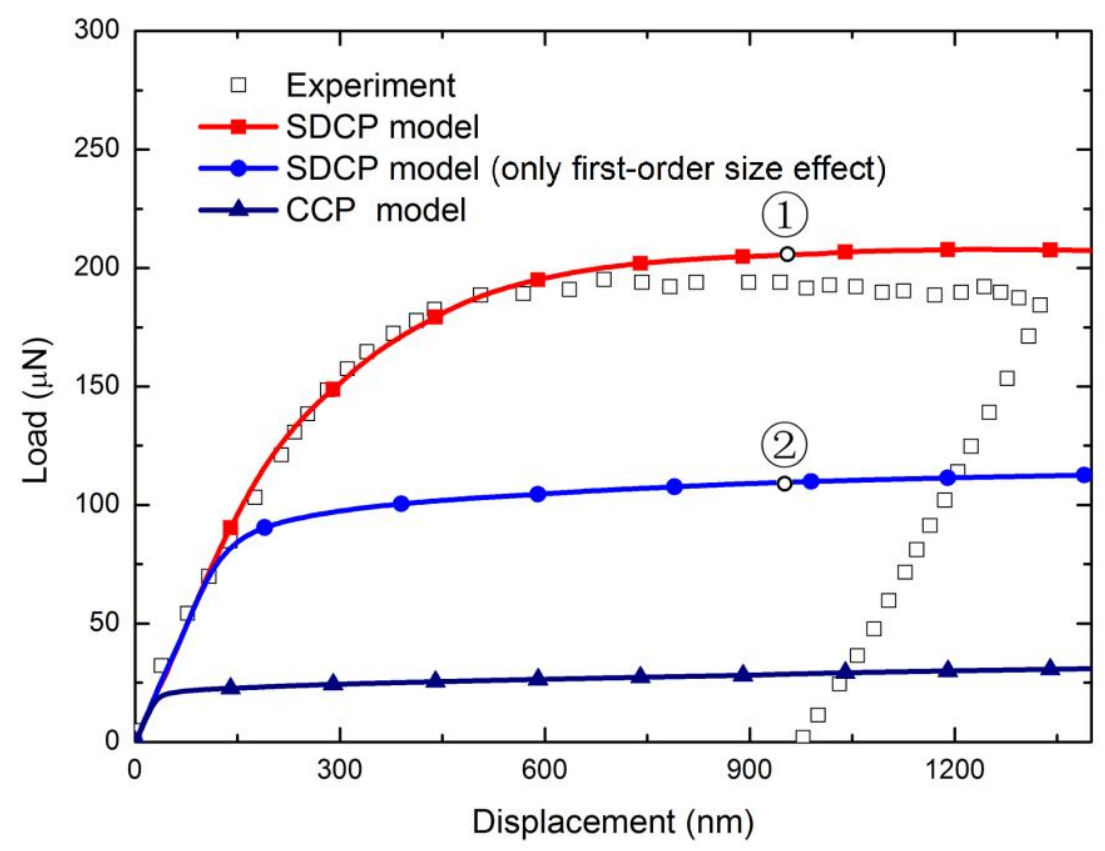

(b)
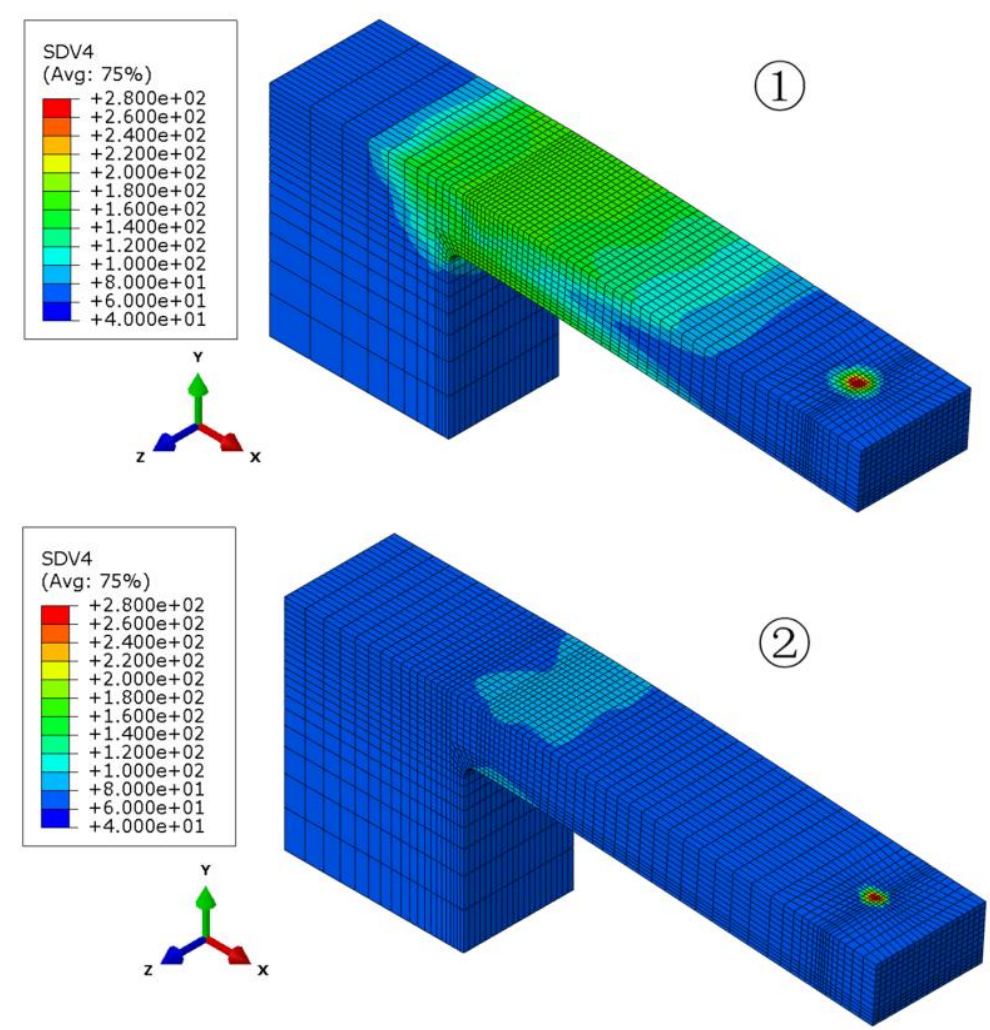

Fig. 7 (a) Comparison of experimental data and simulation results for cantilever beam with dimensions of $5.0 \mu m \times 2.5 \mu m \times 16.3 \mu m$; (b) slip resistance on slip system [$111]<101>$ corresponding to (1) and (2) in Fig. 7(a). 


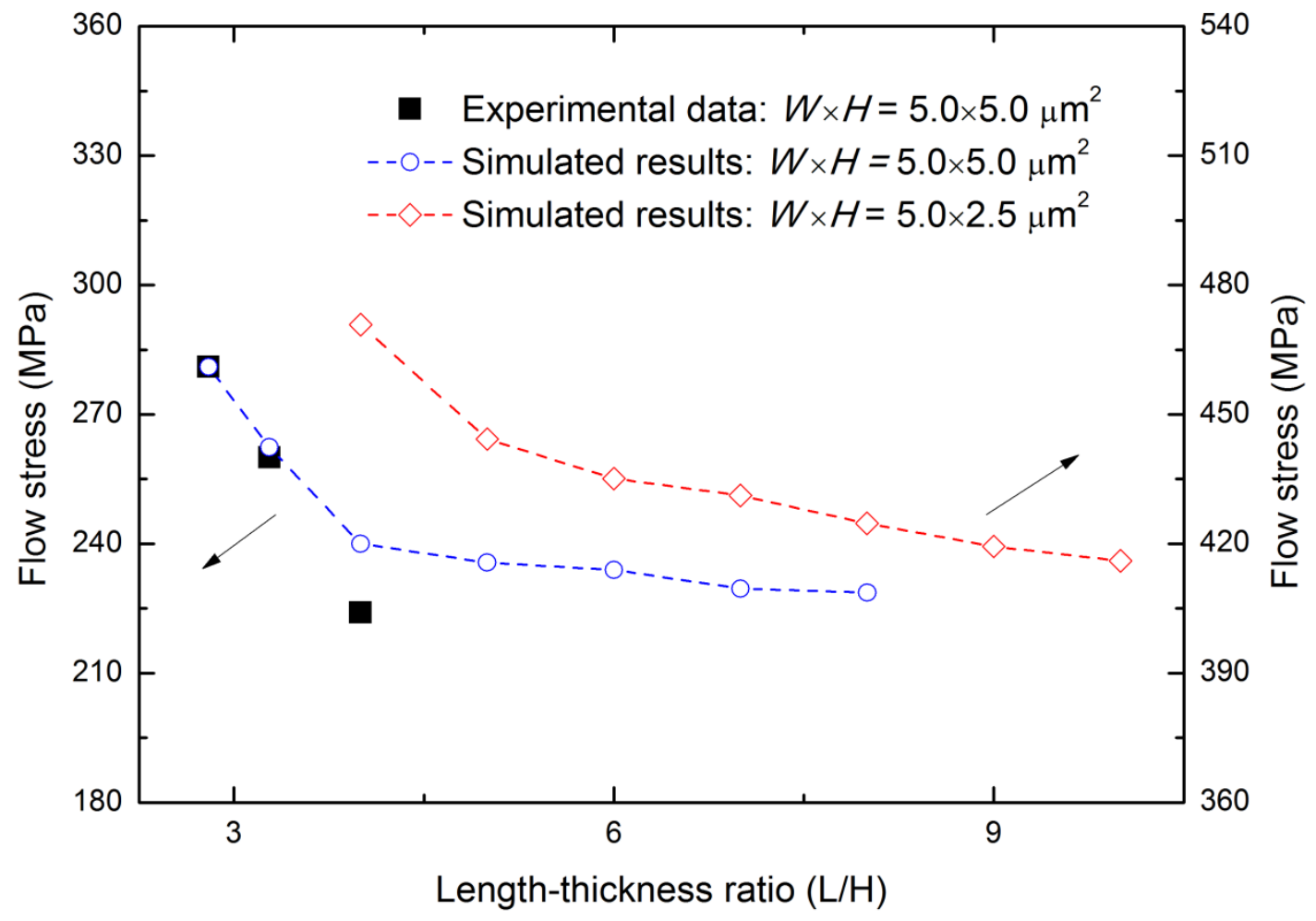

Fig. 8 Effect of geometry on estimation of effective flow stress of cantilever beam

To study the sample-size effect in bending, the effective flow stress (as defined by Eq. (25)) is typically related to the sample size (Gong and Wilkinson, 2011; Motz et al., 2005). The definition of flow stress in Eq. (25) is based on the Euler beam theory, which is dependent on the length-thickness ratio $(L / H)$ of the beam. The range of values of $L / H$ for the beams in the experimental study was limited (Motz et al., 2005). However, our studies indicate that the influence of $L / H$ is substantial and needs to be considered before quantitative estimation of the sample-size effect is carried out. Without loss of the generality, two types of cantilever beams with different cross-sectional geometry are employed to investigate the influence of $L / H$ on the effective flow stress for the specific cross-sectional beams. The numerical results in Fig. 8 show that the effective flow stress decreases with the increasing length-thickness ratio for the two types of cantilever beams. The variation of the effective flowing is large when $L / H<6.0$. The influence of $L / H$ on the calculated flow stress is consistent with the experimental results of Motz et al. (Motz et al., 2005) for the 
cantilever beam with cross-section of $5.0 \mu \mathrm{m} \times 5.0 \mu \mathrm{m}$. Note that all the material parameters are fixed in the parametric study in Fig. 8, and, hence, the influence of $L / H$ is due to the peculiarity of Eq. (25) based on the Euler beam theory. Other experimental studies (Maaß et al., 2015) verify that samples with lower aspect ratios exhibit a more pronounced size effect. Our studies, as shown in Fig. 8, indicate that such a conclusion should be assessed carefully to avoid a spurious determination of phenomena not related to physics of the deformation process.

A comparison of the effective flow stress between the simulation results and the experimental data (Motz et al., 2005) for cantilever beams with different sample sizes is shown in Figure 9. The table (see inset Fig. 9) lists the effective geometrical parameters of cantilever beams used in experiments of Motz et al. (Motz et al., 2005). The simulation results correlate well with the experimental data when the geometrical parameters of the numerical samples are same as the experimental ones. It indicates that the present SDCP model indeed capture the first-order and second-order effects accurately, and the existence of second-order effect determines a more significant sample size effect in bending than that in compression. As indicated by Fig. 8, the effective flow stress of the cantilever beam is estimated incorrectly for a small ratio $L / H$ due to the inaccuracy of Eq. (25). It means that different values of $L / H$ may show different sample size effects. To eliminate such an influence, the simulation results obtained for beams with relatively large and constant $L / H=8.0$ are also presented in Fig. 9. Also, the widely adopted relationship, $\sigma_{f}=\sigma_{0}+K d^{-n}$, is used to fit the simulation results obtained for both variable and constant values of $L / H$, respectively. Here $\sigma_{0}$ is taken as $135 \mathrm{MPa}$, corresponding to the stress at $10 \%$ strain along [110] as shown in Fig. 2. It is clear that there is a difference between the two strategies for quantitative estimation of sample size effect; for example, the exponent $n=1.0$ for the variable $L / H$ while $n=1.3$ for the constant $L / H$. Thus, estimation of the sample 
size effect in bending is more challenging, since it is determined not only by both first-order and second-order effects but also specific features of the sample's geometry.

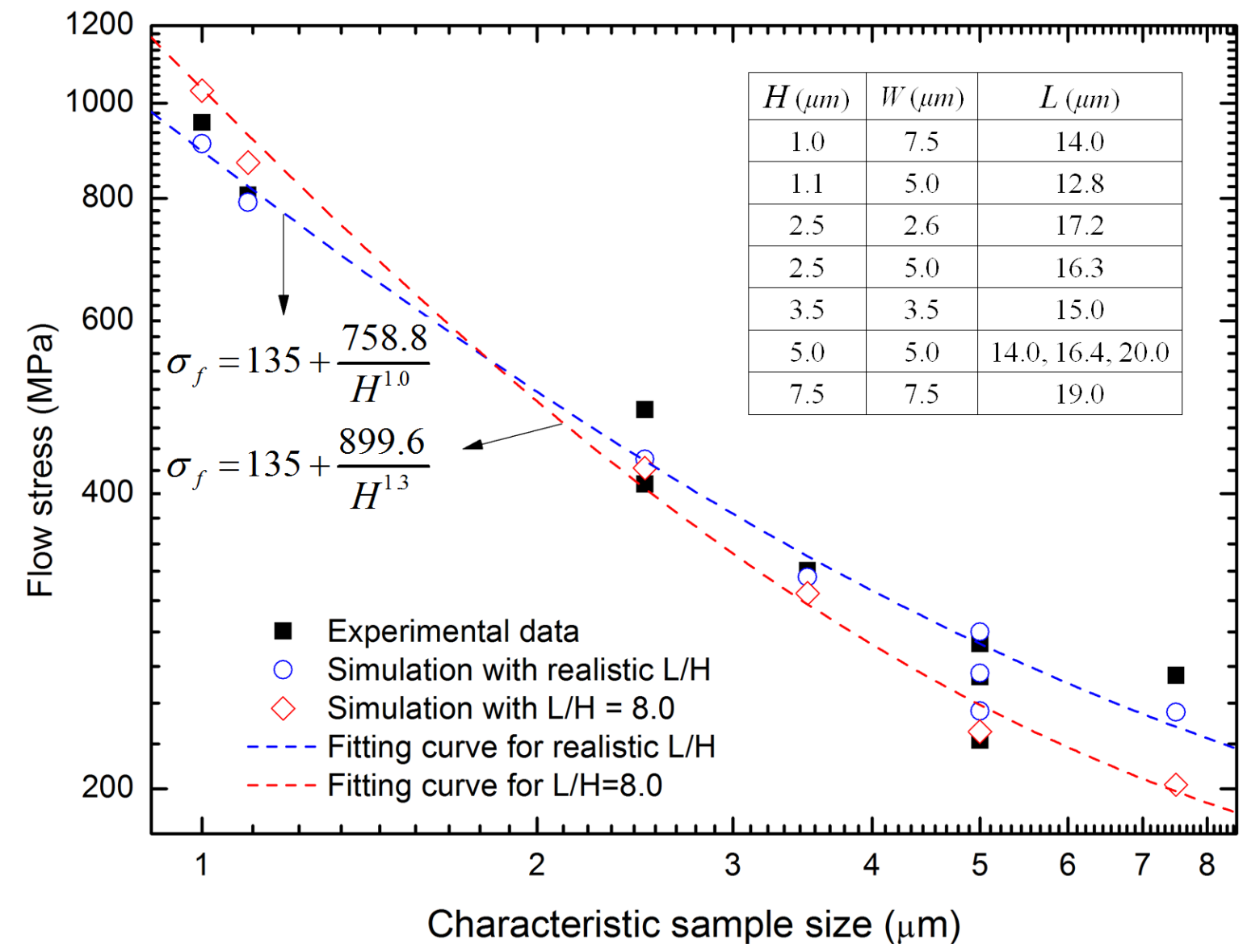

Fig. 9 Sample size effect of $\mathrm{Cu}$ single crystal obtained with cantilever beam: experimental data and simulation results. The table lists the effective geometrical parameters of cantilever beams used in the experiments of Motz et al. (Motz et al., 2005).

\section{Concluding Remarks}

The sample size effect of small-scale specimen of single crystal copper is investigated using the phenomenological SDCP model, in which the contributions from first-order and second-order effects are considered. In particular, the model is capable to capture size effect in both compression and bending deformation regimes. 
The proposed model is a practical extension of a strain-gradient crystal-plasticity formulation, which requires no additional parameters over and above those demanded by the underlying strain-gradient model. The essential factor for modelling accuracy is availability of uniaxial test data capturing the first-order effects and experimental data for the loading states incorporating macroscopically inhomogeneous deformation (such as bending) incorporating influence of both first-order and second-order effects. We are currently exploring the applicability of the modelling framework in capturing size effect in Ti-64 with an HCP crystalline structure.

\section{Acknowledgement}

Funding from the Engineering and Physical Sciences Research Council (UK) through grant EP/K028316/1 and Department of Science and Technology (India), project MAST, is gratefully acknowledged.

\section{References}

Byer, C.M., Ramesh, K., 2013. Effects of the initial dislocation density on size effects in single-crystal magnesium. Acta Materialia 61, 3808-3818.

Demir, E., Raabe, D., 2010. Mechanical and microstructural single-crystal Bauschinger effects: Observation of reversible plasticity in copper during bending. Acta Materialia 58, 6055-6063.

Deshpande, V., Needleman, A., Van der Giessen, E., 2005. Plasticity size effects in tension and compression of single crystals. Journal of the Mechanics and Physics of Solids 53, 26612691.

El-Awady, J.A., 2015. Unravelling the physics of size-dependent dislocation-mediated plasticity. Nature communications 6.

Faghihi, D., Voyiadjis, G.Z., 2012. Determination of nanoindentation size effects and variable material intrinsic length scale for body-centered cubic metals. Mechanics of Materials 44, 189-211.

Geers, M., Brekelmans, W., Janssen, P., 2006. Size effects in miniaturized polycrystalline FCC samples: strengthening versus weakening. International Journal of Solids and Structures 43, 7304-7321.

Gong, J., Wilkinson, A.J., 2011. A microcantilever investigation of size effect, solid-solution strengthening and second-phase strengthening for $<$ a $>$ prism slip in alpha-Ti. Acta Materialia 59, 5970-5981. 
Greer, J.R., De Hosson, J.T.M., 2011. Plasticity in small-sized metallic systems: Intrinsic versus extrinsic size effect. Progress in Materials Science 56, 654-724.

Huang, Y., 1991. A User-material Subroutine Incroporating Single Crystal Plasticity in the ABAQUS Finite Element Program. Harvard Univ.

Huang, Y., Zhang, F., Hwang, K., Nix, W., Pharr, G., Feng, G., 2006. A model of size effects in nano-indentation. Journal of the Mechanics and Physics of Solids 54, 1668-1686.

Hug, E., Dubos, P., Keller, C., Duchêne, L., Habraken, A., 2015. Size effects and temperature dependence on strain-hardening mechanisms in some face centered cubic materials. Mechanics of Materials 91, 136-151.

Hutchinson, J., 1976. Bounds and self-consistent estimates for creep of polycrystalline materials. Proceedings of the Royal Society of London. A. Mathematical and Physical Sciences 348, 101-127.

Kalidindi, S.R., Anand, L., 1993. Large deformation simple compression of a copper single crystal. Metallurgical and Materials Transactions A 24, 989-992.

Kiener, D., Guruprasad, P., Keralavarma, S., Dehm, G., Benzerga, A., 2011. Work hardening in micropillar compression: In situ experiments and modeling. Acta Materialia 59, 3825-3840. Kiener, D., Motz, C., Dehm, G., 2009. Micro-compression testing: A critical discussion of experimental constraints. Materials Science and Engineering: A 505, 79-87.

Kiener, D., Motz, C., Schöberl, T., Jenko, M., Dehm, G., 2006. Determination of mechanical properties of copper at the micron scale. Advanced Engineering Materials 8, 1119-1125.

Ma, A., Roters, F., Raabe, D., 2006. A dislocation density based constitutive model for crystal plasticity FEM including geometrically necessary dislocations. Acta Materialia 54, 2169-2179.

Maass, R., Van Petegem, S., Ma, D., Zimmermann, J., Grolimund, D., Roters, F., Van Swygenhoven, H., Raabe, D., 2009. Smaller is stronger: The effect of strain hardening. Acta Materialia 57, 5996-6005.

Maaß, R., Volkert, C., Derlet, P., 2015. Crystal size effect in two dimensions-Influence of size and shape. Scripta Materialia 102, 27-30.

Motz, C., Schöberl, T., Pippan, R., 2005. Mechanical properties of micro-sized copper bending beams machined by the focused ion beam technique. Acta Materialia 53, 4269-4279.

Parthasarathy, T.A., Rao, S.I., Dimiduk, D.M., Uchic, M.D., Trinkle, D.R., 2007. Contribution to size effect of yield strength from the stochastics of dislocation source lengths in finite samples. Scripta Materialia 56, 313-316.

Raabe, D., Ma, D., Roters, F., 2007. Effects of initial orientation, sample geometry and friction on anisotropy and crystallographic orientation changes in single crystal microcompression deformation: A crystal plasticity finite element study. Acta Materialia 55, 4567-4583.

Reuber, C., Eisenlohr, P., Roters, F., Raabe, D., 2014. Dislocation density distribution around an indent in single-crystalline nickel: Comparing nonlocal crystal plasticity finite-element predictions with experiments. Acta Materialia 71, 333-348.

Roters, F., Eisenlohr, P., Hantcherli, L., Tjahjanto, D., Bieler, T., Raabe, D., 2010. Overview of constitutive laws, kinematics, homogenization and multiscale methods in crystal plasticity finite-element modeling: Theory, experiments, applications. Acta Materialia 58, 1152-1211.

Sun, Q., Guo, Q., Yao, X., Xiao, L., Greer, J.R., Sun, J., 2011. Size effects in strength and plasticity of single-crystalline titanium micropillars with prismatic slip orientation. Scripta Materialia 65, 473-476.

Takeuchi, T., 1975. Work hardening of copper single crystals with multiple glide orientations. Transactions of the Japan Institute of Metals 16, 629-640.

Tang, H., Schwarz, K., Espinosa, H., 2007. Dislocation escape-related size effects in singlecrystal micropillars under uniaxial compression. Acta Materialia 55, 1607-1616. 
Tarleton, E., Balint, D., Gong, J., Wilkinson, A., 2015. A discrete dislocation plasticity study of the micro-cantilever size effect. Acta Materialia 88, 271-282.

van Beers, P., Kouznetsova, V., Geers, M., 2015. Grain boundary interfacial plasticity with incorporation of internal structure and energy. Mechanics of Materials 90, 69-82.

Ye, J., Mishra, R.K., Sachdev, A.K., Minor, A.M., 2011. In situ TEM compression testing of Mg and Mg-0.2 wt.\% Ce single crystals. Scripta Materialia 64, 292-295. 